\title{
Cell Junction
}

National Cancer Institute

\section{Source}

National Cancer Institute. Cell Junction. NCI Thesaurus. Code C13983.

Cell junction is the specialized focal contact between cell-cell or cell-matrix in all tissues.

Cell junctions have various functions either communication, anchoring or org anization.

There are specific protein associated with subtype of cell junctions and most of the junctions are too small to be resolved by light microscope. 